\title{
Autonomous dry stone
}

\section{On-site planning and assembly of stone walls with a robotic excavator}

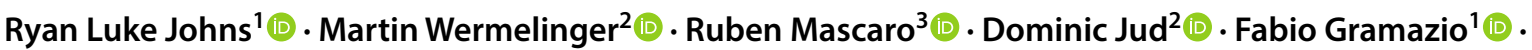 \\ Matthias Kohler ${ }^{1}\left(\right.$ ) Margarita $\mathrm{Chli}^{3} \odot \cdot$ Marco Hutter $^{2} \odot$
}

Received: 15 June 2020 / Accepted: 9 September 2020 / Published online: 29 September 2020

(c) The Author(s) 2020

\begin{abstract}
On-site robotic construction not only has the potential to enable architectural assemblies that exceed the size and complexity practical with laboratory-based prefabrication methods, but also offers the opportunity to leverage context-specific, locally sourced materials that are inexpensive, abundant, and low in embodied energy. We introduce a process for constructing dry stone walls in situ, facilitated by a customized autonomous hydraulic excavator. Cabin-mounted LiDAR sensors provide for terrain mapping, stone localization and digitization, and a planning algorithm determines the placement position of each stone. As the properties of the materials are unknown at the beginning of construction, and because error propagation can hinder the efficacy of pre-planned assemblies with non-uniform components, the structure is planned on-the-fly: the desired position of each stone is computed immediately before it is placed, and any settling or unexpected deviations are accounted for. We present the first result of this geometric- and motion-planning process: a 3-m-tall wall composed of 40 stones with an average weight of $760 \mathrm{~kg}$.
\end{abstract}

Keywords On site robotics · Dry stone walls · Adaptive assembly

Ryan Luke Johns

johns@arch.ethz.ch

$\bowtie$ Martin Wermelinger

martiwer@mavt.ethz.ch

Ruben Mascaro

rmascaro@ethz.ch

Dominic Jud

dominic.jud@mavt.ethz.ch

Fabio Gramazio

gramazio@arch.ethz.ch

Matthias Kohler

kohler@arch.ethz.ch

Margarita Chli

chlim@ethz.ch

Marco Hutter

mahutter@ethz.ch

1 Gramazio Kohler Research, ETH Zurich, Zurich, Switzerland

2 Robotic Systems Lab, ETH Zurich, Zurich, Switzerland

3 Vision for Robotics Lab, ETH Zurich, Zurich, Switzerland

\section{Introduction}

Computational planning and fabrication tools have the potential to greatly increase the sustainability of architectural construction, allowing for the use of abundantly available natural and reclaimed materials that are currently too complex or time-intensive for cost-effective widespread application. As compared to many common building materials, minimally processed rock and locally recycled demolition debris have extremely low embodied energy (Morel et al. 2001; Alcorn 2003), but also have the potential to express site-specific materiality, revive regional vernacular building methods, and exhibit new forms of "cultural performance" (Oesterle 2009).

This chapter presents an integrated system for constructing double-faced dry stone walls in situ using a customized autonomous mobile hydraulic excavator. We outline a process for mapping the environment, and localizing and digitizing irregular stones. Using this digitized information, we algorithmically determine the position and orientation of stones to align with a designer-indicated goal surface, and conduct grasp- and motion planning for collision-free 


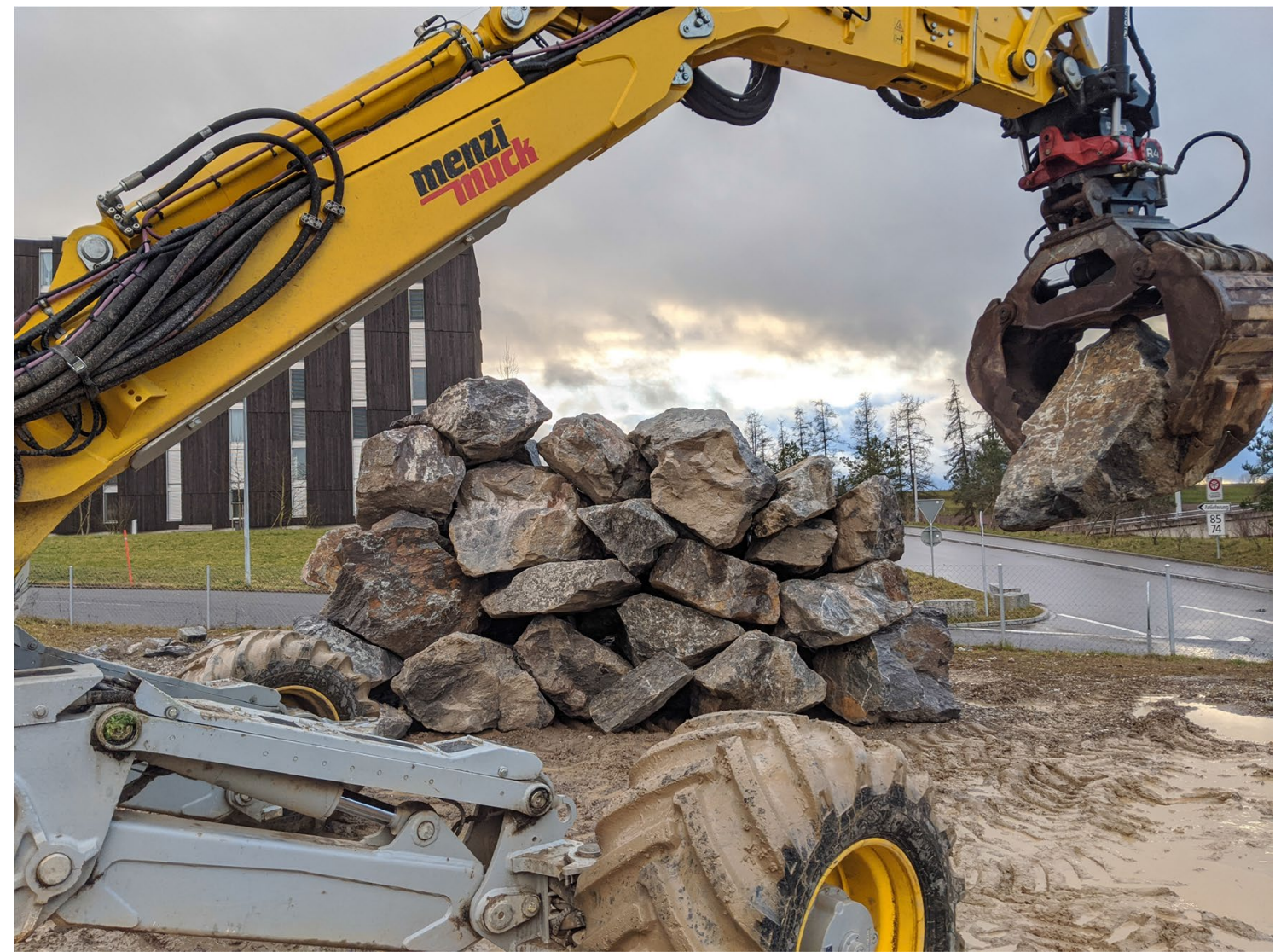

Fig. 1 Stone wall constructed with autonomous hydraulic excavator

placement. We demonstrate the applicability of our method by constructing a 3-m-tall wall with a total length of $5 \mathrm{~m}$ out of 40 stones with masses between 230 and $1548 \mathrm{~kg}$ (Fig. 1).

\section{Related work}

A number of recent projects in the domain of architectural digital fabrication have made use of nonstandard input materials combined with robotic construction. Such projects have played a substantial role in re-establishing computational design and fabrication as a practice that can not only materialize digital complexity, but also understand and adapt to the existing complexity of the material world. Johns et al. (2018) provide an overview of recent projects in this general domain, while a number of notable projects deal more specifically with irregular stone-based assembly: "Smart Scrap" uses automotive digitization tools to inventory partially dressed limestone for facade planning (De Boer et al. 2007), while "Cyclopean Cannibalism" revives ancient polygonal masonry methods through robotic stone cutting (Clifford et al. 2018). The latter fits parallel-faced polygonal elements within the constraints of demolition concrete and stone offcuts, using $73 \%$ of the stock material (selected from a larger set) after dressing. In comparison, the work presented in this chapter makes use of $100 \%$ of each stone object, and uses every stone in the available set.

Toward the assembly of such raw materials, the geometric nesting of unmodified parts has been explored in two dimensions with simulated annealing (Lambert and Kennedy 2012). Others have demonstrated the potential of shape descriptors for matching 3D shapes to existing geometries for the generation of 3D collages without constraints of fabricability (Gal et al. 2007), or for the reassembly of fractured objects without structural constraints (Huang et al. 2006).

The demonstrators presented by Furrer et al. (2017) and Liu et al. (2019) both perform dry stacking of unmodified stones, making use of stationary robotic manipulators and RGB-D scanners for the localization of pre-scanned stones. Both projects consider structural stability using a physics simulator and evaluate a cost function to determine the validity of each potential pose. The former is focused on the construction of vertical stacks, while the latter can build one-layer thick walls of up to four courses with a $40 \%$ success rate in an open loop fashion (without accounting for the settling of placed stones). In this work, we overcome the 
design limitations of such one- and two-dimensional structures, presenting a planner capable of building a multiplelayer-thick structure following an arbitrarily specified target geometry. Stones are scanned in situ on the fly, and the as-built wall is monitored to refine the placed-stone poses before the planner is executed again.
Several projects have been developed which demonstrate the potential of large-scale autonomous construction with on site robotics. Generally combining mobile platforms with conventional industrial robots, these compound setups have been predominantly demonstrated with standardized building components (Dörfler et al. 2016) or industrially produced chemical products (Keating et al. 2014). Recent research has
Fig. 2 Setup diagram: A available stones, B constructed wall, C search space, D rim path with keypoints, E grid map, F gripped stone, G LiDAR sensors, H GNSS antennas, I network antenna, J HEAP platform with axes indicated, $\mathrm{K}$ reference scale and payload comparison with human and industrial robot

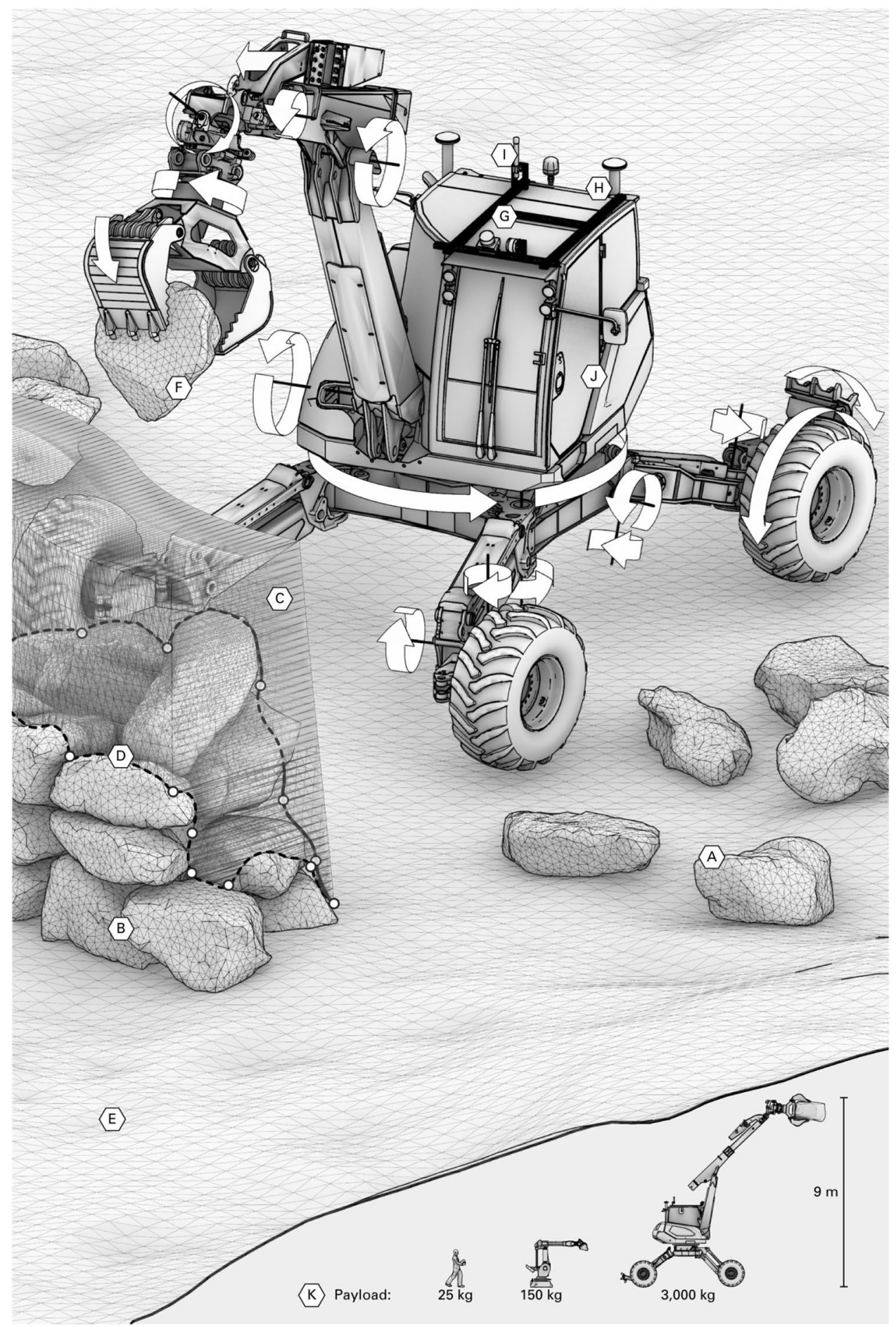


demonstrated the potential of robotic systems to construct auxiliary structures to achieve and maintain navigability in previously unknown or untraversable terrain, but these experiments have only been demonstrated at a small scale, and do not make use of naturally occurring found construction materials (like stones), but instead use compliant bags (Saboia et al. 2019) or polyurethane foam (Fujisawa et al. 2015) to homogenize the irregularities in their environment.

\section{Methodology}

\subsection{Platform}

The stone wall was created using HEAP (Hydraulic Excavator for an Autonomous Purpose), a highly customized Menzi Muck M545 12t walking excavator that was developed for autonomous applications and advanced teleoperation (Fig. 2J). The fully mobile 23-axis excavator is capable of lifting objects to a height of $9 \mathrm{~m}$ and can freely manipulate items weighing up to $3000 \mathrm{~kg}$. Adaptable to complex terrain, the machine is equipped with force-controllable hydraulic cylinders that allow for control of ground interaction forces, and cabin- and chassis-mounted inertial measurement units (IMUs) that track machine orientation. Global cabin localization is achieved by a Leica iCON iXE3 with two global navigation satellite system (GNSS) antennas and a receiver (Fig. 2h). Real-time kinematic (RTK) corrections for the GNSS signals are received over the network from permanently installed base stations for improved accuracy. Draw wire encoders are used to measure the position and velocity of the hydraulic arm- and grapple cylinders.

For exteroceptive sensing (scanning of the environment, available stones, and in-progress structure), two Velodyne Puck VLP-16 LiDAR scanners are placed at the front edge of the cabin's roof to provide 3D scans. The LiDARs are mounted orthogonally to each other, such that scan points accumulate both while driving the robot around and while pivoting the cabin (Fig. $2 \mathrm{~g}$ ). For a more detailed description of the system's sensors and actuators, we refer the reader to Jud et al. (2019).

All software components of the project are written in $\mathrm{C}++$, and the robot operating system (ROS) is used to transfer data over the network between the different software nodes distributed on several computers. The ROS master, managing the connection between processes, is located on the on-board computer of HEAP.

\subsection{Stone localization and scanning}

To reconstruct and localize the stones in the surroundings of the excavator, we use the LiDARs mounted on the cabin roof
(Fig. 2g). Before the construction process begins, the available stones are loosely distributed on the terrain (Fig. 3). This facilitates the detection of single stone instances by first segmenting the ground plane from the LiDAR map, and subsequently performing Euclidean clustering on the remaining point cloud to distinguish the single objects. These point clusters are used to compute an initial grasp configuration on the not yet reconstructed stone segments. Once a given stone is picked up using this relatively low-resolution data, a high-resolution scan of the full stone is completed while it is held in the gripper. In the following sections, we describe this stone reconstruction process, the LiDAR-based scene mapping, and the object pose refinement based on the reconstruction and scene map information.

Reconstruction For the 3D reconstruction, the end effector is spun with a continuous velocity in front of the LiDAR sensors while holding the stone. With the known geometry of the gripper, we filter out points that belong to the end effector while accumulating points on the stone. The complete contour of the stone is recorded by concatenating multiple point clouds from different viewing angles, and applying Poisson surface reconstruction (Kazhdan et al. 2006) to generate the surface mesh from the point cloud data.

LiDAR-based scene mapping A precise map of the excavator's complete surrounding (including the as-built structure) is necessary for planning grasp configurations and arm motions, and for facilitating the decision of where and how to position stones. However, simple accumulation of single LiDAR scans is very temporally and spatially limited, as precise knowledge of the sensor's motion is necessary (and error accumulation leads to drift over time). To create a consistent map over large time- and motion intervals, we use LaserSLAM (Dubé et al. 2017), a laser-based graph SLAM that considers the odometry constraints provided by the excavator's state estimation, fusing GNSS measurements with the IMUs, and scan matching constraints provided by registering a new scan pair using the Iterative Closest Point (ICP) algorithm with a map consisting of the previous scans. Self-see points of the excavator's arm or legs in the LiDAR scans are filtered to get a 3D point cloud, built locally around the current robot location, without being corrupted by its moving parts (Fig. 7a).

ICP refinement Being able to refine the pose of an already reconstructed stone in the environment (Fig. 7b) or in the gripper is an essential capability, as the stone might settle during placement or shift as it is being picked up. For this pose refinement, we first sample a point cloud on the reconstructed mesh of the stone. Using an ICP step, this point cloud is registered to the scene map (if the stone is placed in the environment), or to the accumulated gripper 
Fig. 3 Initial state of stones, loosely distributed on the terrain. LiDAR mapping and RANSAC ground segmentation allow for initial detection and grasping for refined scanning in the gripper

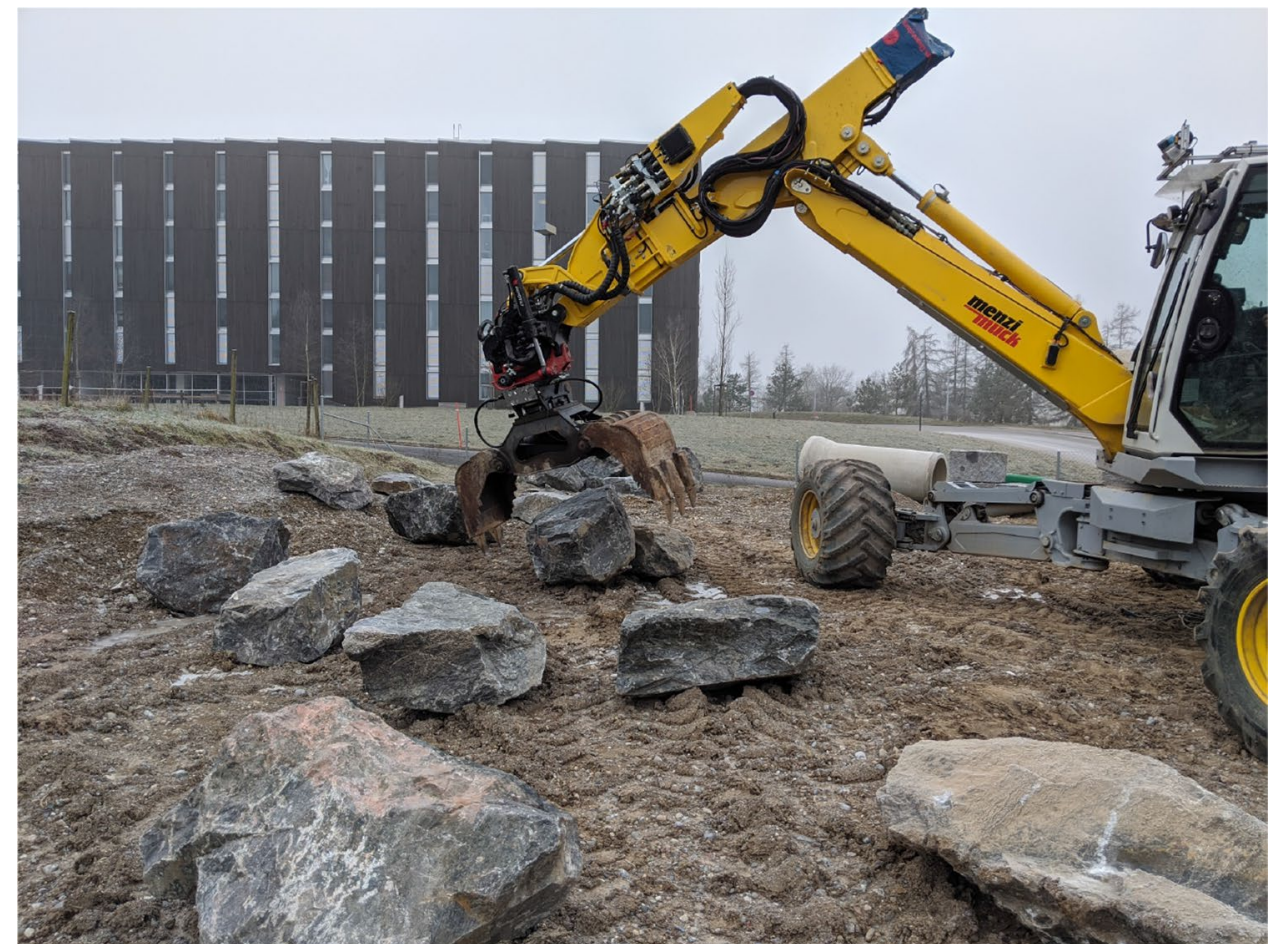

cloud (if it is grasped by the excavator). To improve the localization, we first segment the ground plane in the vicinity of the stone using RANSAC-based 3D plane fitting. Additional filtering is necessary, however, as stones might be cluttered and partially occluded, especially if they are constituents of the in-progress wall. Therefore, the scene map is further segmented using Euclidean clustering, and segments belonging to already refined stones or segments too far from the assumed pose are removed before the ICP step. This allows for the reliable pose refinement of objects even if they are partially occluded by the built structure. If the initial orientation of the stone is unknown (e.g. after it has been overturned), the ICP step is performed for multiple initial orientations and the refined transformation with the highest ICP-score is selected.

\subsection{Geometric planning}

The iterative selection, positioning and orienting of each stone is determined by a geometric planning software that attempts to construct a double-faced wall bounded vertically by any user-specified mesh target surface, and below by the LiDAR-scanned elevation map of the existing terrain. Given any number of available stones, the software determines the preferred placement from the available solutions, and the selected stone and the desired transformation are further processed for grasp planning and physical positioning. Rather than pre-planning the entire wall, the software computes solutions on the fly, stone by stone. This is done for two reasons:

First, for sufficiently large constructions, it is impractical to pre-scan, sort, and store hundreds or thousands of stones when the required space and complexity can instead be minimized if they are salvaged, unearthed, or delivered ondemand, as needed. Computing solutions on the fly allows for a more adaptive construction that is not held up if one pre-planned stone goes missing.

Second, the absolute rigidity of stone makes for significant error propagation that would result in undesirable deviation from the desired geometry, and higher probability of structural collapse in turn. Any minute difference in the scanned geometry can cause unexpected collisions or settling during placement, and the current setup allows for these changes to be automatically accounted for in the next solution.

Finding a suitable position of even one stone in a relatively small area can be a computationally heavy problem, and the irregular nature of the stones and substrate geometry make it generally difficult to apply stochastic solvers or continuous optimization methods. The software should generally find a valid solution in less time than it takes for the excavator to physically locate, grasp, and place a stone, such that the finding of solutions does not significantly deter progress (at present, the solver takes approximately $1 \mathrm{~min}$ per stone on a laptop running Ubuntu 18.04 with an Intel i7-8750H 4.1 GHz Processor and 16GB RAM). 

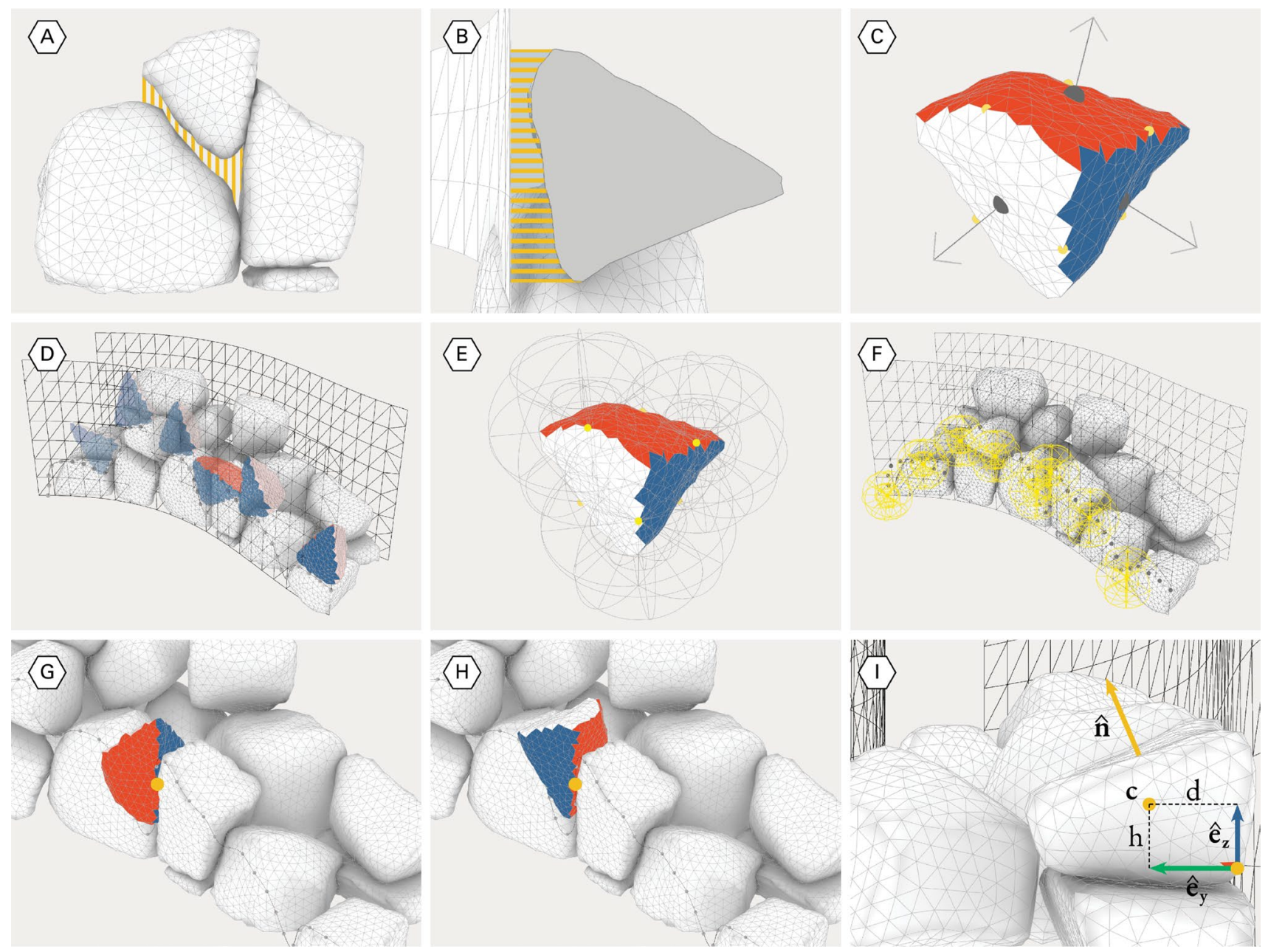

Fig. 4 Stone solver goals and methods. a Minimize volume under stone, b minimize volume between stone face and goal surface, c compute faces and edges, d sample along rim path, e compute

Much like the algorithm presented by Liu et al. (2019), we make use of a number of heuristics derived from conventional stone masonry techniques to reduce the size of the solution space. However, our approach differs significantly in the specification and sequencing of these heuristics: we use both the geometric properties of the stones and the constraints of the designer-specified goal surface to inform an algorithm that begins with fast computational checks, and increases in complexity as the solution space is substantially reduced.

For any given stone placement, the following high level goals are established: determine a structurally stable solution that minimizes the volume directly under the stone in question (Fig. 4a) and also minimizes the volume between the exposed stone face and the designated geometry of the wall surface (Fig. 4b). To achieve these objectives, we implement the following subroutines: descriptors at edge midpoints, $\mathbf{f}$ descriptors at rim path points, $\mathbf{g}, \mathbf{h}$ two possible orientations for each partial match, $\mathbf{i}$ stability and upper normal direction factors

Stone attributes At all times, the software holds an inventory of stones that have been scanned. Upon receipt of new information from the scanning node, the stone mesh is immediately cleaned by removing any degenerate faces and disjoint remnants that might remain from the Poisson reconstruction. The mass properties are computed using the method described by Bacher et al. (2014), and each stone is repositioned such that its centroid is at the origin, and its principal axes (computed using PCA on the mesh vertices) are aligned with the global frame. An iterative implementation of the variational shape approximation method (CohenSteiner et al. 2004) is used to determine approximate partitioned face surfaces on the stones given an allowable normal deviation per clustered region of mesh triangles. From these partitioned regions, a face-adjacency matrix is generated, stone edges and edge midpoints are identified, and the mean weighted normal of each region is used to represent the normal direction of each partitioned face surface (Fig. 4c). 
Search space A volumetric search space (Fig. 2c) is defined as the region bounded by the designated outer wall surfaces on all sides and above, and bounded below by the ground and the upper surface of the in-progress wall. While this upper surface is in reality generally composed of many disjointed stones, it is approximated as a single continuous surface using a simulated LiDAR scanner on the known mesh data of the ground and placed stones, and meshed using Poisson reconstruction. Thus, both the empty ground and the upper bounds of a half-built wall are treated similarly as the foundation for the next stone. The intersection between this reconstructed "ground" surface and the target wall face surfaces defines the rim path, which we identify as the upper perimeter edge of the structure at any given state (the contour where the top surface of the as-built structure intersects with the vertical goal wall surfaces)(Fig. 2d).

An even sampling along the rim path serves as a onedimensional list of possible placement positions, and this list is further reduced to only include lower points within a given distance of identified keypoints (Fig. 2d) along this path: keypoints are defined as any point along the rim path that lies either between two stones, where one stone meets the ground, or at the corners or ends of designated structure. This filtering process ensures that the wall is generally built up evenly, and reduces the likelihood of running joints that weaken the bonding of the wall.

Fit estimation Given the known edge midpoints, edge directions, and face-normal vectors on the stones, and the normal vectors of the goal wall surface at each point on the rim path, it is trivial to determine the transformations necessary to place any specified stone on this path-such that a given stone edge is tangent to the rim path, and the stone face is aligned with the wall surface. While each combination of path point and stone edge could be sampled (Fig. 4d), this still represents a substantially large solution space if the wall is long and there are multiple known stones in the available inventory. Just as a stone mason would likely only try placing stones that look like they will fit (instead of brute force sampling every possible position), we first check if a stone is geometrically similar to the region in question using rotationally invariant shape descriptors before attempting any more involved processing (Figs. 4e, f, 5). Specifically, we use the FPFH descriptor (Rusu et al. 2009) at each edge midpoint and at each rim path vertex coupled with a k-nearest neighbor (KNN) search to find the $k$-closest geometric matches at each included point on the rim path $(k \approx 10)$. In this demonstrator, the KNN search typically reduced over a million possible combinations of stone edges with rim path points to some thousands. Because of the rotationally invariant nature of such shape descriptors, each match must consider both possible orientations of an edge with the rim path (Figs. 4g, 4h).

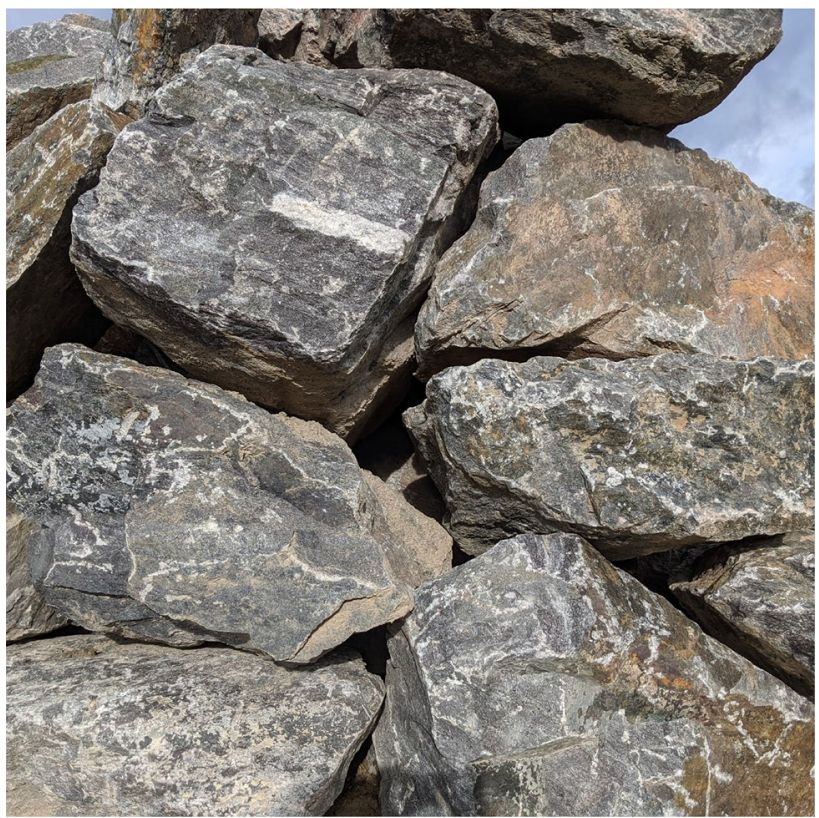

Fig. 5 Elevation detail of the constructed wall. Rotationally invariant shape descriptors allow for a tight fit by initially matching available stone surfaces to corresponding negative geometry of the wall

Stability heuristics Prior to running any involved physics simulations, we remove potential candidates that are likely unstable, or likely to reduce future stability, informed by guidance from stone masonry literature. In dry stone walls, it is generally advised to not place stones in such an orientation that their thinnest dimension defines the depth into the wall-thus acting as a thin veneer that is more likely to fall away from the wall (Environmental Action Foundation 2019). We generalize this requirement as needing to meet a minimum ratio between the horizontal $(d)$ and vertical $(h)$ distance measured between the specified point on the rim path and the stone centroid (Fig. 4i):

$d / h>0.5$.

As the construction process uses a double-faced method, the structure benefits from having an inward slope at the top of each placed stone-such that any settling is minimized and supported by stones on the opposing side of the wall (Vivian 1976). ${ }^{1}$ For each intended position, we take an even sampling of the exposed stone surface using a raycasting method, and compute the mean top-exposed surface normal

\footnotetext{
${ }^{1}$ Note that this inward slope differs from the inward slope employed by Liu et al. (2019): in our use and in the masonry literature, the inward slope is a sectional property of a double faced wall. Liu et al. use it to mean a lowered center of mass between the end stones of a given bond, as observed across the front elevation (thereby creating a sagging bond in a single-layered structure).
} 


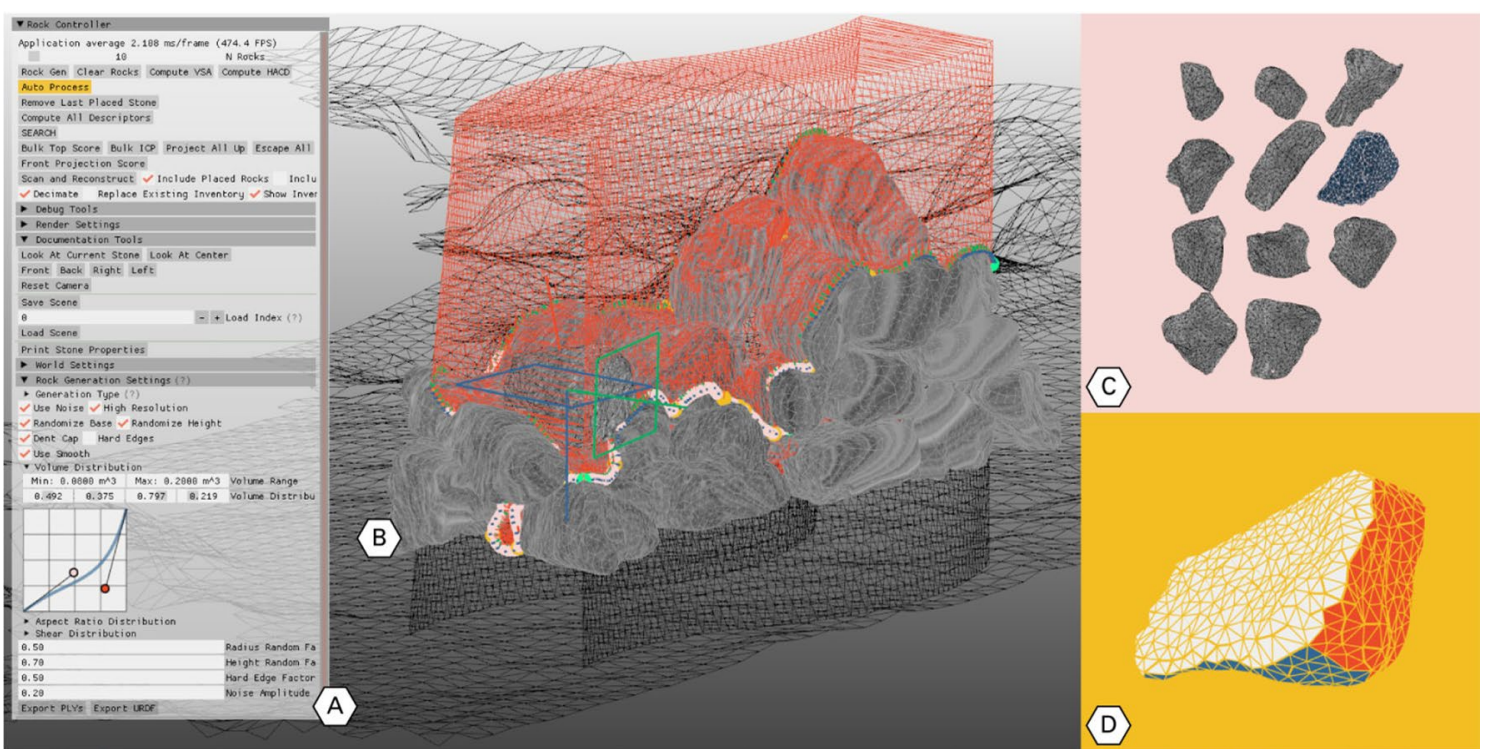

Fig. 6 Screen capture of geometric planning software. a Imgui Interface, $\mathbf{b}$ active environment with search space, solutions, and placed stones, $\mathbf{c}$ available scanned stones in inventory, $\mathbf{d}$ selected stone viewer with VSA faces

Fig. 7 Grasp planning and placement of a stone to its desired location (red). A Collision and localization point cloud, B stones localized in point cloud, $\mathrm{C}$ spline motion trajectory, (D, green) collision-free grasp hypotheses, (E, red) grasp hypotheses with collisions, $\mathrm{F}$ selected grasp configuration. $\mathrm{G}$ The collision point cloud is augmented with point clouds of already localized stones

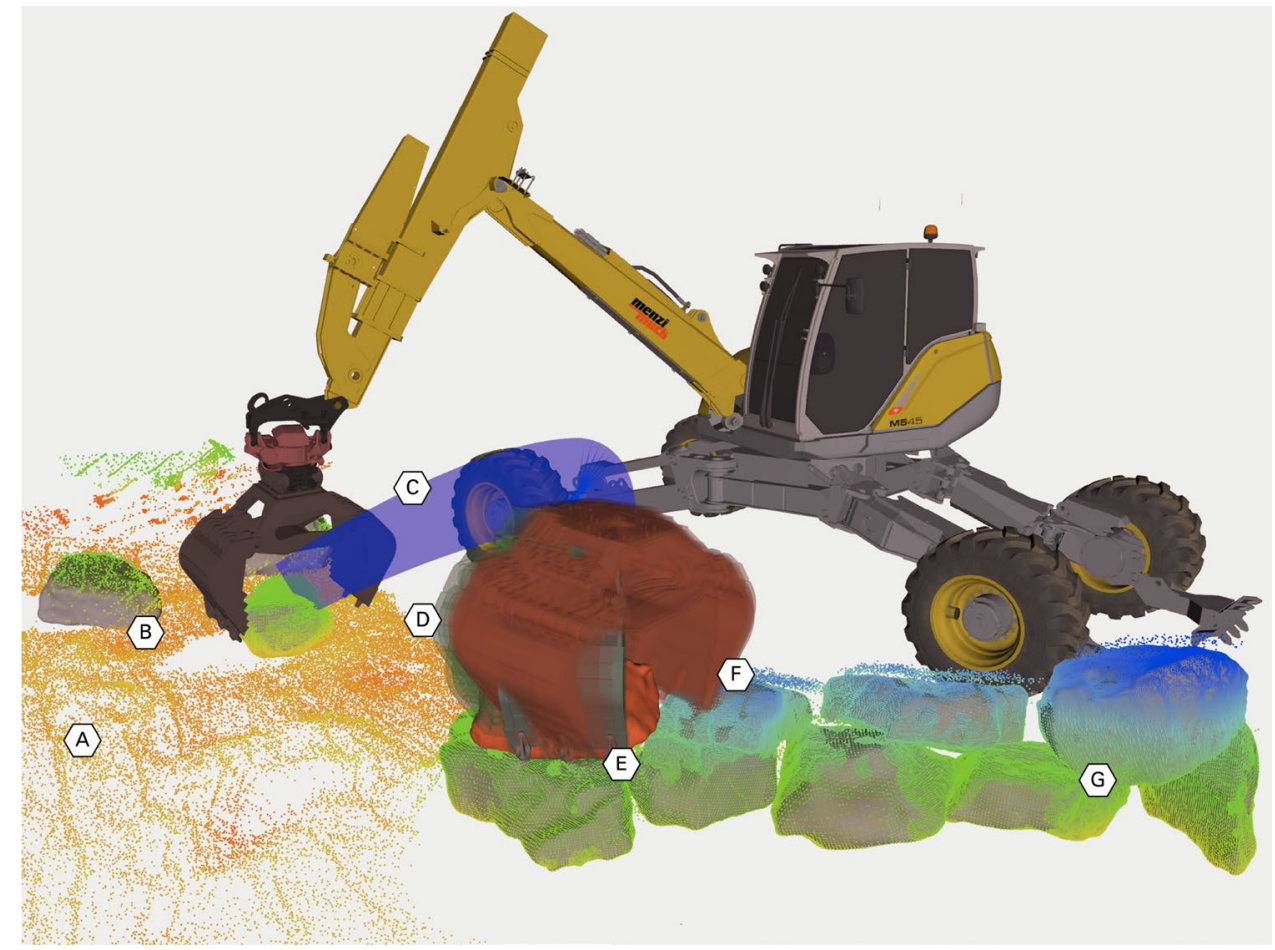

(î) from the stone normal direction at each hit point. We then verify that $\hat{\mathbf{n}}$ points into the wall and that the stone surface is not excessively steep by

$\hat{\mathbf{e}}_{\mathbf{y}} \cdot \hat{\mathbf{n}}>=0$ and $\hat{\mathbf{e}}_{\mathbf{z}} \cdot \hat{\mathbf{n}}>0.7$, where $\hat{\mathbf{e}}_{\mathbf{y}}$ is the unitized inward-facing surface normal of the target wall surface projected onto the horizontal plane, and $\hat{\mathbf{e}}_{\mathbf{z}}$ is a unit vector pointing against the direction of gravity (Fig. 4I). 
ICP refinement Once the pool of potential matches has been reduced by the aforementioned heuristics, each candidate is refined using ICP to better align it with the surrounding stones and goal wall surface.

Physics Following ICP refinement, the remaining solutions are sorted by their ICP score, and (if specified) reduced to a preset maximum number of solutions. These are then simulated for stability with the Bullet physics engine (Coumans 2015), and solutions that do not reach equilibrium within a fixed simulated-world-time period or within a distance moved are ruled out.

Scoring The remaining stones after the physics simulation step are sorted using the combined parameters from Fig. 4a and $b$, where the gap volume is calculated with a raycasting technique measuring the displacement of hits measured from local plan- and elevation projection planes with and without the placed stone (and considering the known stone volume). The best match is then sent for physical placement. A provided Imgui interface (Cornut 2005) allows for alternative high-scoring solutions to be selected manually, if desired.

The geometric planning software makes extensive use of libigl (Jacobson et al. 2018) for mesh processing, PCL for point cloud operations (Rusu and Cousins 2011), and openframeworks (Lieberman et al. 2005) for visuals (Fig. 6).

\subsection{Grasp and placement execution}

The goal of the grasp pose planning is to find viable grasping configurations that allow the excavator to pick a desired stone and place it at the planned location without collisions with the existing wall (Fig. 7e). A grasp configuration is defined as 6-DoF pose of the excavator's gripper, where contact with the desired stone can be performed. To find a viable grasp configuration that respects the collision constraints, we sample a large amount of grasp hypotheses on the stone of interest in the map point cloud. Those grasp hypotheses are validated for collisions by intersecting a polyhedral gripper model with the map cloud and searching for inliers. Note that both the grasp configuration and the corresponding placement configuration must be verified to be without collisions (Fig. 7d, f). Due to occlusions, the map point cloud may contain holes that can result in undetected collisions. To prevent these situations, the map point cloud is augmented with point clouds generated from the meshes of already localized objects (Fig. 7g). After ruling out colliding configurations, the remaining grasp poses are evaluated for force closure and scored by task-specific criteria (alignment to ground, grasp encompassment, and distance to stone CoM) to obtain the final grasp.

A motion planner is used to generate a spline trajectory for reaching the desired grasp pose and for moving from the grasp pose to the placement pose (Fig. 7c). The approach direction during placement is chosen by raycasting to provide the largest margin to obstacles. Figure 7 shows the spline trajectory (c, blue) for moving the grasped stone from the ground to its desired placement pose (e, red). Each time a stone is grasped or released, its pose with respect to the map or end effector is updated with an ICP refinement step using its previous location as the initial guess (Sect. 3.2).

\section{Experiment}

The localization, scanning, and geometric- and motionplanning routines developed in this work were used to construct a large-scale (3-m-high and 5-m-long) test wall using 40 available gneiss stones ${ }^{2}$ with an average mass of 757 $\mathrm{kg}$ (range: $230-1584 \mathrm{~kg}$ ). To our knowledge, it represents both the greatest height and number of components of any masonry structure autonomously constructed from irregular stones. It is essential to note, however, that such qualifications are heavily influenced by the geometry of the available material, and one might much more easily construct a manybonded wall with ashlar-like stones. Despite the relatively large size of the available stones, ${ }^{3}$ the outer face of the asbuilt structure was measured to be generally within $10 \mathrm{~cm}$ of the intended target surface (Fig. 8). Figure 9 shows the side elevation of the completed structure, and the front elevation can be seen in Fig. 1.

While the geometric planner allows for solutions to be found with any number of stones in the inventory, it predictably finds better correspondences if there are more stones to choose from (at the computational expense of an increased search space). For a much larger construction, one would likely scan and build in batches, keeping tens of digitized stones in the inventory, and refilling it when no solution can be found within some tolerance requirements. For this experiment, there was a fixed number of available stones (40), and they were each scanned and added to the digital inventory before the construction of wall commenced. This accelerated construction and provided a wide variety of stones for the initial planning steps. The planning and execution were performed sequentially, meaning that after each placement the stone pose was refined and updated in the geometric planning before initiating the next update step. Figure 10 shows the intermediate steps of the wall construction with 2 and 20 placed stones.

\footnotetext{
${ }^{2}$ Gneiss is a locally abundant metamorphic rock often used for architectural applications, landscape construction, and retaining walls.

3 These are classified as 'medium-coarse boulders' on the modified Udden-Wentworth grain-size scale (Blair and McPherson 1999).
} 
Fig. 8 Digital model of constructed wall with goal surfaces and indicated distance between placed stones and one target surface

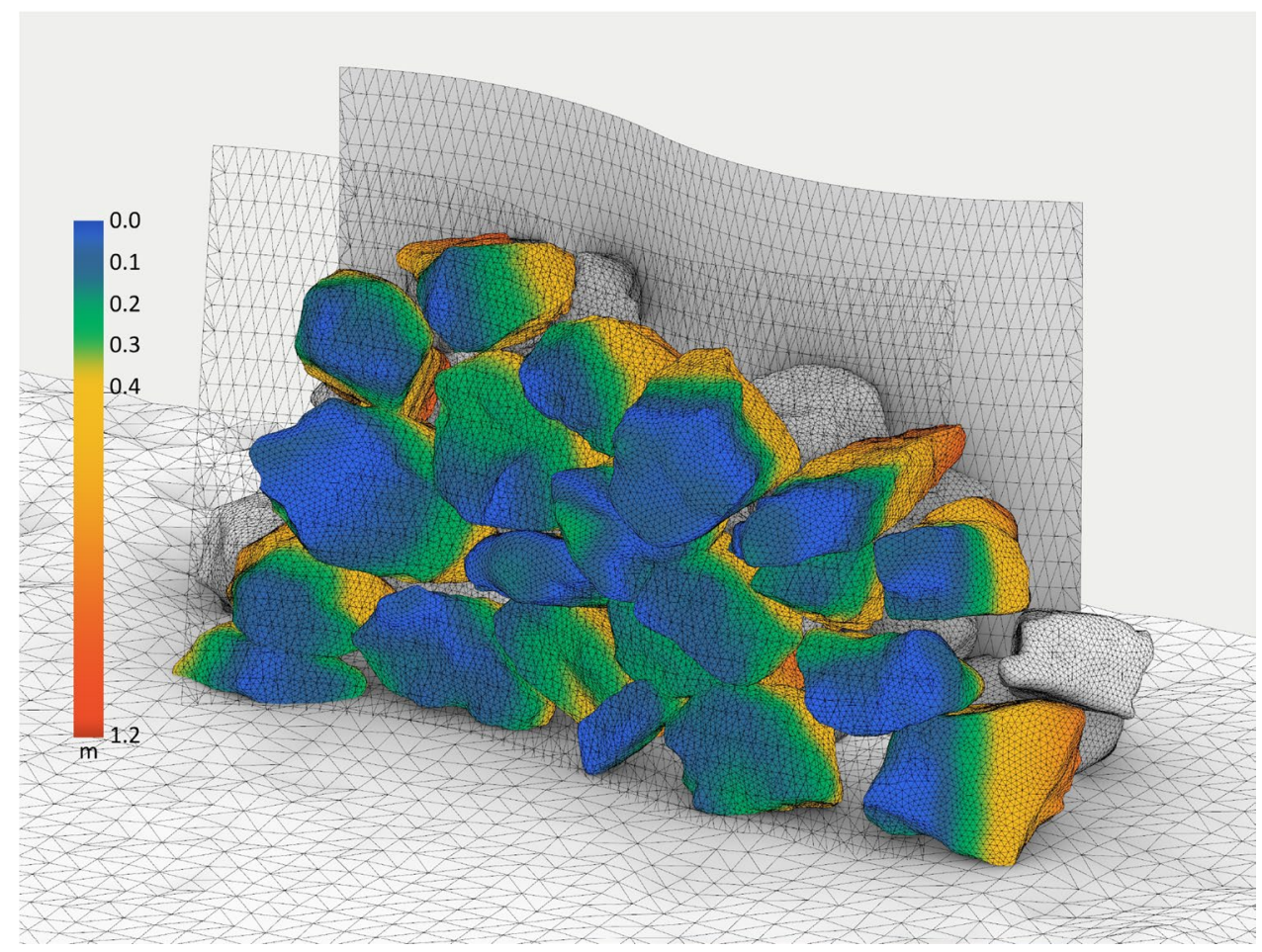

\section{Improvements}

A number of improvements are intended which will provide increased process robustness and allow for additional design constraints to influence the solver. While this initial prototype was imagined as a section of a longer wall (Figs. 11, 12), future developments will resolve the particularities of terminating details at wall ends and corners: the use of alternating stretchers and headers with returned faces that both strengthen the structure and clearly define the arris (edge where surfaces meet) (Cramb 1992).

One major limitation of the setup at present is that we have not yet implemented the autonomous flipping of stones. On the occasion that placement solutions are found which cannot be grasped in the current stone position, an alert is generated that conveys the need for manual intervention: the stone must be flipped manually before the autonomous setup will relocalize the stone and continue construction. The drawback of using LiDARs for reconstruction and ICP refinement is the need to always move the sensor to accumulate scans into the complete 3D model: this is time consuming and renders the current sensors ill-equipped for dynamic tracking during stone flipping. This task will be made more feasible with the imminent integration of additional sensors, including RGB cameras. By texturing the reconstruction of the stones (Waechter et al. 2014), it is possible to extract visual features that can be used for recognizing a stone instance or

Fig. 9 Side elevation of constructed wall conveying double-faced structure and near-vertical orientation 
Fig. 10 Wall construction in progress with 2 (above) and 20 (below) placed stones out of the 40 used in this experiment
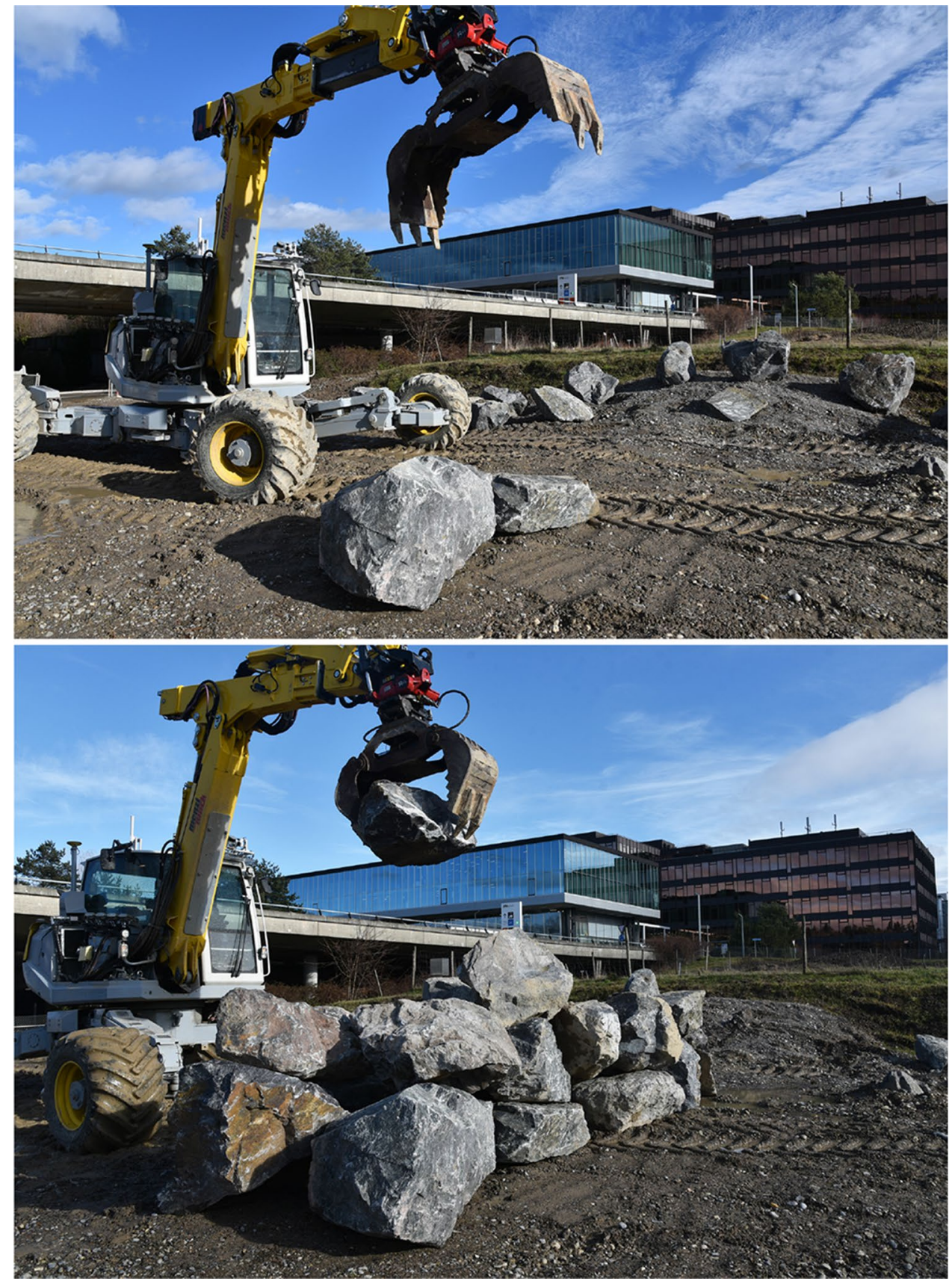

tracking its motion (Marchand et al. 2016). This will not only better facilitate dynamic tasks such as flipping, but could also be considered as a component in the design process. Provided that a structure contained enough stones to make a pattern legible, it would be possible to incorporate variable distributions of color, scale, or surface shapes to meet additional design goals beyond the global shape of the wall.

Whereas we currently rely on physics simulation for stability assessment, in future work we will consider structural validation by physically probing the as-built construction. The excavator-equipped with pressure sensors and an additional force-torque sensor-would serve as both a construction tool and testing device, using applied loads to verify the stability of the structure and measure deformations.

\section{Discussion}

This research demonstrates a robotically enabled process for creating structures from a wide range of input and target geometries: allowing digital design goals to 
Fig. 11 In progress wall with overlay of potential extension of structure, generated by allowing the solver to continue with additional digital stone models
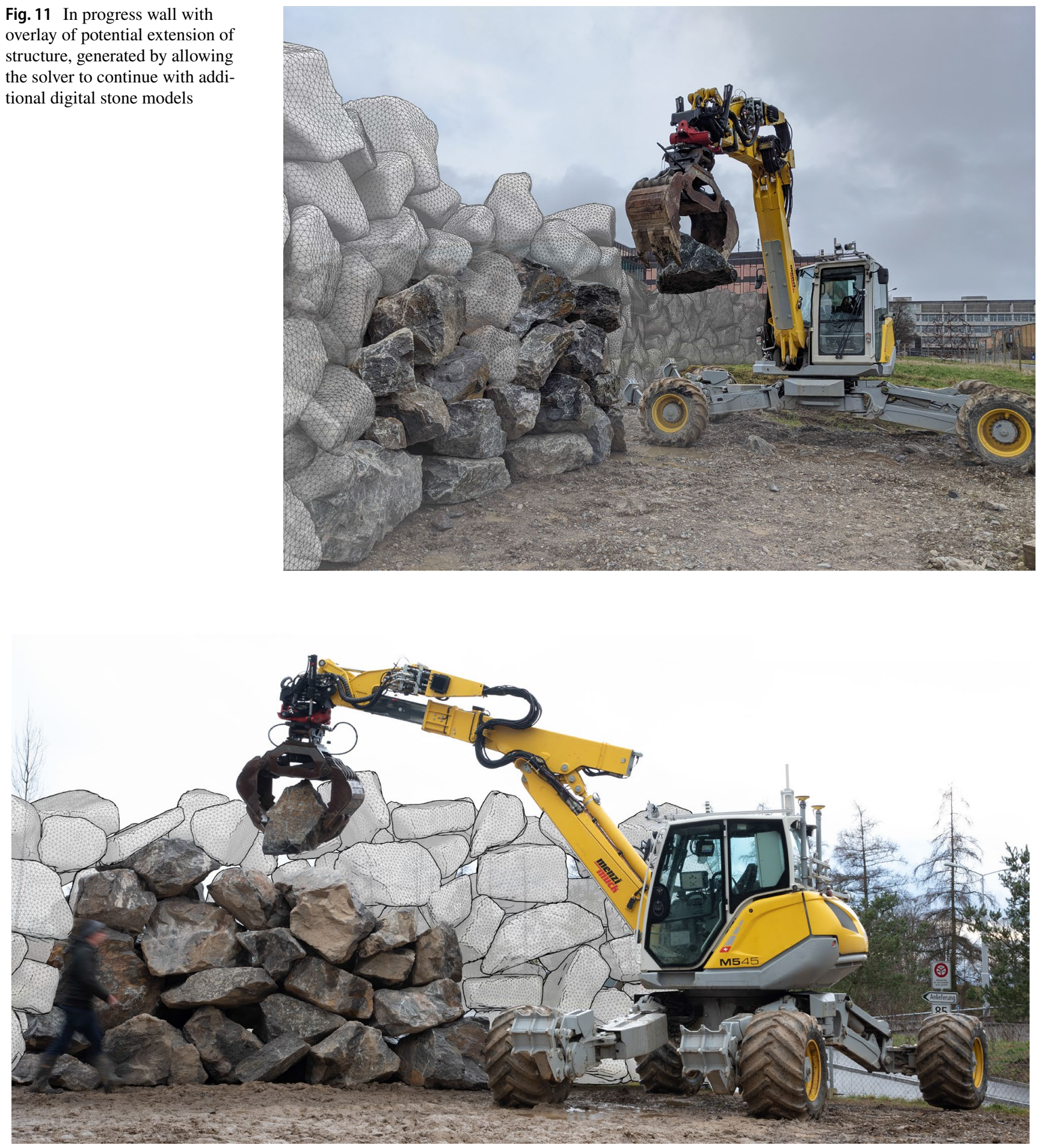

Fig. 12 Constructed wall with figure (left) for scale and indicated potential extensions

be integrated with existing ancient construction methods for double-faced freestanding dry stone walls built from unprocessed or minimally processed natural stone. In contrast to cut stone (and even more so to concrete), these exhibit extremely low embodied energy because there is minimal transportation required ${ }^{4}$, and because they do not require energy-intensive cutting or post-processing.

${ }^{4}$ For this experiment, the raw stones were delivered by truck from a quarry located $40 \mathrm{~km}$ from the test field. 
While the embodied energy of stone construction varies regionally, post-processing (sawing, etc.) is a major contributor to the overall environmental impact of masonry architecture: despite an increase in the thickness of the built structure when compared to cut stone, building with raw stone can be environmentally advantageous (Ioannidou et al. 2014).

Rather than modifying individual stones, this method focuses on cleanly fitting the exposed surfaces of stones along the outer two faces of a given wall section, while tolerating necessary gaps between stones within the hidden space of the poché. Even in a well-constructed dry stone wall, these gaps can account for $20-40 \%$ of the constructed volume (Villemus 2004; Mundell 2009), and are essential in enabling the use of completely irregular and indeterminate source materials: a dry stone wall is thus "defined by the spaces between the stones as much as it is by the stones themselves" (Snow 2008). While such gaps can be filled in with smaller 'chinking' stones (Vivian 1976) or with mortar, they can also be left as supplemental habitats for flora and fauna to thrive (Witschi 2019).

Dry stone construction has diminished in popularity in the last century, despite the environmental, economic, and aesthetic advantages of building with inexpensive, local, and natural materials: increasing labor costs combined with comparatively inexpensive and simple-to-install mass manufactured building components have rendered irregular stone construction infeasible in many situations (Bätzing 2019; Ioannidou et al. 2018). By synthesizing knowledge from manual dry stone masonry handbooks (Vivian 1976; Cramb 1992; Environmental Action Foundation 2019) together with new methods of computational design and robotic construction, this work aspires to reactivate irregular stone construction while enabling new modes of design expression and large-scale fabrication.

\section{Conclusion}

In this chapter, we introduce a platform and algorithm for the autonomous construction of large-scale dry stone walls in situ, with the aid of a customized robotic excavator-addressing the challenge of using unprocessed and locally available construction material. The addition of a high-payload, fully mobile platform has greatly expanded the 'machinic morphospace' (Menges 2013) of robotic construction tools on site, allowing us in turn to better navigate the constrained 'material morphospace' of complex and nonstandard found objects (Johns and Foley 2014). The process has potential to greatly reduce the embodied energy of architectural construction in specific contexts, and can be extended to work with a range of source materials, including reclaimed demolition debris. By realizing a double-faced wall on an architectural scale, we demonstrate the applicability of automated stone masonry for future construction processes.

Acknowledgements This research was supported by the Swiss National Science Foundation through the National Centre of Competence in Digital Fabrication (NCCR dfab). Gramazio Kohler Research postdoctoral support: Kathrin Dörfler, Aleksandra Anna Apolinarska, Lauren Vasey.

Funding Open access funding provided by Swiss Federal Institute of Technology Zurich.

Open Access This article is licensed under a Creative Commons Attribution 4.0 International License, which permits use, sharing, adaptation, distribution and reproduction in any medium or format, as long as you give appropriate credit to the original author(s) and the source, provide a link to the Creative Commons licence, and indicate if changes were made. The images or other third party material in this article are included in the article's Creative Commons licence, unless indicated otherwise in a credit line to the material. If material is not included in the article's Creative Commons licence and your intended use is not permitted by statutory regulation or exceeds the permitted use, you will need to obtain permission directly from the copyright holder. To view a copy of this licence, visit http://creativecommons.org/licenses/by/4.0/.

\section{References}

Alcorn A (2003) Embodied energy and $\mathrm{CO}_{2}$ coefficients for NZ building materials. Centre for Building Performance Research,

Bacher M et al (2014) Mass properties of triangulated solids and their derivatives, supplemental material for 'spin-it: optimizing moment of inertia for spinnable objects'. ACM Trans Graph (TOG) 33(4):2

Bätzing W (2019) Life in the Alps-is there a future? In: by Environmental Action Foundation (eds) Dry stone walls: basics, construction, significance, 1st edn. Scheidegger \& Spiess, Zurich, pp. 379-383

Blair TC, McPherson JG (1999) Grain-size and textural classification of coarse sedimentary particles. J Sedi Res 69(1):6-19

Clifford B, McGee W, Muhonen M (2018) Recovering cannibalism in architecture with a return to cyclopean masonry. Nexus Netw J 20(3):583-604

Cohen-Steiner D, Alliez P, Desbrun M (2004) Variational shape approximation. ACM Trans Graph (TOG) 23(3):905-914

Cornut O (2005) Dear ImGui: bloat-free immediate mode graphical user interface for $\mathrm{C}++$ with minimal dependencies. https://githu b.com/ocornut/imgui Accessed 11 Jun 2020

Coumans E (2015) Bullet physics simulation. In: ACM SIGGRAPH 2015 Courses. SIGGRAPH '15. Association for Computing Machinery, Los Angeles, California, p. 1

Cramb I (1992) The art of the stonemason. Alan C. Hood \& Co, Chamersburg, $\mathrm{p} 174$

De Boer J et al (2007) Smart scrap. http://i-m-a-d-e.org/?p=2201. Accessed 3 Jul 2020

Dörfler K et al (2016) Mobile robotic brickwork. In: Reinhardt D, Saunders R, Burry J (eds) Robotic fabrication in architecture, art and design 2016. Springer International Publishing, Cham, pp 204-217

Dubé R et al (2017) An online multi-robot SLAM system for 3D LiDARs. In: 2017 IEEE/RSJ international conference on intelligent robots and systems (IROS), pp. 1004-1011 
Environmental Action Foundation (ed) (2019) Dry stone walls: basics, construction. Significance. Scheidegger \& Spiess, Zurich, p 472

Fujisawa $\mathrm{R}$ et al (2015) Active modification of the environment by a robot with construction abilities. ROBOMECH J 2(1):9

Furrer F et al (2017) Autonomous robotic stone stacking with online next best object target pose planning. In: 2017 IEEE international conference on robotics and automation (ICRA), pp. 2350-2356

Gal R et al (2007) 3D collage: expressive non-realistic modeling. In: Proceedings of the 5th international symposium on non-photorealistic animation and rendering. NPAR '07. San Diego, California: Association for Computing Machinery, pp. 7-14

Huang Q-X et al (2006) Reassembling fractured objects by geometric matching. ACM Trans. Graph. 25(3):569-578

Ioannidou $\mathrm{D}$ et al (2018) Where does the money go? Economic flow analysis of construction projects. Build Res Inf 46(4):348-366

Ioannidou D, Zerbi S, Habert G (2014) When more is better-comparative LCA of wall systems with stone. Build Environ 82:628-639

Jacobson A, Panozzo D et al (2018) Libigl: a simple C++ Geometry Processing Library. https://libigl.github.io/

Johns RL, Anderson JS (2018) Interfaces for adaptive assembly. In: ACADIA // 2018: Recalibration. On imprecisionand infidelity. 38th annual conference of the association for computer aided design in architecture. CUMINCAD, Mexico City, pp. 126-135

Johns RL, Foley N (2014) Bandsawn bands. In: McGee W, Ponce de Leon M (eds) Robotic fabrication in architecture, art and design 2014. Springer International Publishing, Cham, pp 17-32

Jud D et al (2019) Autonomous free-form trenching using a walking excavator. IEEE Robot Automat Lett 4(4):3208-3215

Kazhdan M, Bolitho M, Hoppe H (2006) Poisson surface reconstruction. In: Proceedings of the fourth eurographics symposium on geometry processing. SGP '06. Cagliari, Sardinia, Italy: Eurographics Association, pp. 61-70

Keating S et al (2014) A compound arm approach to digital construction. In: McGee W, Ponce de Leon M (eds) Robotic Fabrication in architecture, art and design 2014. Springer International Publishing, Cham, pp 99-110

Lambert M, Kennedy P (2012) Using artificial intelligence to build with unprocessed rock. Key Eng Mater 517:939-945

Lieberman Z et al (2005) openFrameworks. https://openframeworks. cc/. Accessed 11 Jun 2020

Liu Y, Choi J, Napp N (2019) Planning for robotic dry stacking with irregular stones. In: Easy-Chair Preprint no. 14531453

Marchand E, Uchiyama H, Spindler F (2016) Pose estimation for augmented reality: a hands-on survey. IEEE Trans Vis Comput Graph 22(12):2633-2651
Menges A (2013) Morphospaces of robotic fabrication. In: Rob-Arch 2012. Springer, Vienna, pp 28-47

Morel JC et al (2001) Building houses with local materials: means to drastically reduce the environmental impact of construction. Build Environ 36(10):1119-1126

Mundell C (2009) Large scale testing of drystone retaining structures. Doctoral Thesis, Department of Architecture \& Civil Engineering, University of Bath

Oesterle S (2009) Cultural performance in robotic timber construction. In: de Estree Sterk T, Loveridge R, Pancoast D (eds) reForm( )-building a better tomorrow: Proceedings of the 29th annual conference of the association for computer aided design in architecture (ACADIA 09). ACADIA, Chicago, IL, pp 194-200

Rusu RB, Blodow N, Beetz M (2009) Fast Point Feature Histograms (FPFH) for 3D Registration. In: 2009 IEEE international conference on robotics and automation, pp. 3212-3217

Rusu RB, Cousins S (2011) 3D is here: point cloud library (PCL). In: 2011 IEEE international conference on robotics and automation, pp. 1-4

Saboia M, Thangavelu V, Napp N (2019) Autonomous multi-material construction with a heterogeneous robot team. Robot and Auton Syst 121:103239

Snow D (2008) Listening to stone: hardy structures, perilous follies, and other tangles with nature. Artisan, New York, p 145

Villemus B (2004) Etude des murs de soutènement en maçonnerie de pierres sèches. Doctoral Thesis, Civil Engineering. L'institut National Des Sciences Appliquees De Lyon, Lyon, France, 254 pp

Vivian J (1976) Building stone walls. Storey Publishing LLC, USA, p 114

Waechter M, Moehrle N, Goesele M (2014) Let there be color! Largescale texturing of 3D reconstructions. In: Fleet D et al (eds) Computer Vision-ECCV 2014. Lecture notes in computer science. Springer International Publishing, Cham, pp 836-850

Witschi F (2019) Flora, fauna. In: Environmental Action Foundation (ed) Dry stone walls: basics, construction, significance, 1 st ed. Scheidegger \& Spiess, Zurich, Switzerland, pp. 329-378

Publisher's Note Springer Nature remains neutral with regard to jurisdictional claims in published maps and institutional affiliations. 